\title{
Breast cancer surgeon is struck off because of risk to patients
}

The $B M J$ reported that a GMC fitness to practise panel had determined that the breast surgeon Miss Puvaneswary Markandoo should be struck off the medical register because her performance was judged "a threat to patients" (BMJ

2011;343:d7249, doi:10.1136/bmj.d7249). Miss Markandoo has asked us to point out that she is appealing against the panel's decision.

Cite this as: BMJ 2011;343:d8285

๑ BMJ Publishing Group Ltd 2011 